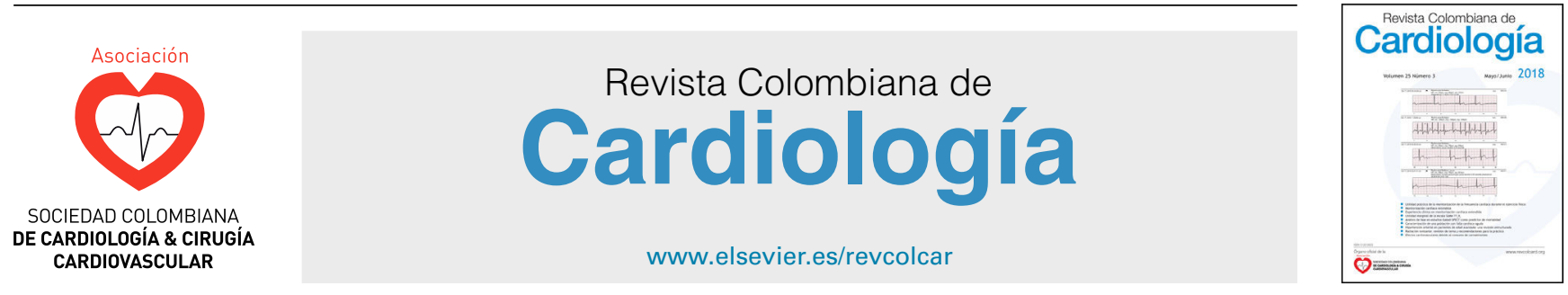

CARDIOLOGÍA DEL ADULTO - PRESENTACIÓN DE CASOS

\title{
Mielitis isquémica como presentación de aorta shaggy en paciente con síndrome aórtico agudo concomitante
}

\section{Carolina Ocampo-Salgado, Mauricio Duque-Ramírez*, Sebastián Naranjo-Restrepo y María del Mar Serna-Posada}

\author{
Departamento de Cardiología y Electrofisiología, Universidad CES, Medellín, Colombia
}

Recibido el 10 de julio de 2018; aceptado el 9 de noviembre de 2018

Disponible en Internet el 8 de abril de 2019

\section{PALABRAS CLAVE \\ Aorta shaggy; \\ Aterosclerosis; \\ Embolia; \\ Embolia visceral; \\ Mielitis}

\section{KEYWORDS}

Shaggy aorta;

Atherosclerosis;

Embolisms;

Visceral embolisms;

Myelitis

\begin{abstract}
Resumen La aorta shaggy se define como una degeneración ateromatosa agresiva y extensa de la aorta, cuya friabilidad predispone a ulceración y complicaciones embólicas, cursa con alto riesgo de embolia sistémica y no se conocen prevalencia ni incidencia en poblaciones de riesgo. La mayoría de casos publicados hacen referencia a complicaciones en procedimientos quirúrgicos. En la literatura no se hallaron reportes que asocien ateroembolia aórtica con obstrucción de la arteria de Adamkiewicz, cuyo diagnóstico no siempre es posible visualizando su oclusión por angiotomografía o por angiorresonancia, pues el defecto puede ser evanescente o puede existir compromiso distal con obstrucción microvascular, difícilmente aparente con arteriografía selectiva.

Se presenta un caso de mielopatía isquémica embólica asociada a aorta shaggy con probable oclusión de la arteria de Adamkiewicz como responsable del deterioro neurológico agudo de la paciente, confirmado por resonancia magnética nuclear.

Para caracterizar mejor esta enfermedad y para tener las estrategias diagnósticas y terapéuticas apropiadas en su abordaje oportuno, consideramos importante el reporte de casos similares aumentando así su sospecha diagnóstica.

(C) 2019 Sociedad Colombiana de Cardiología y Cirugía Cardiovascular. Publicado por Elsevier España, S.L.U. Este es un artículo Open Access bajo la licencia CC BY-NC-ND (http:// creativecommons.org/licenses/by-nc-nd/4.0/).
\end{abstract}

Ischaemic myelitis as a presentation of a shaggy aorta in a patient with concomitant acute aortic syndrome

Abstract A shaggy aorta is defined as an aggressive and extensive atheromatous degeneration of the aorta. Its friability predisposes to ulceration and embolic complications. It carries a high risk of systemic embolisms, and its prevalence and incidence in risk populations is unknown. The majority of published cases mention complications in surgical procedures. No reports have been found in the literature that associate aortic atheroembolism with obstruction of the

\footnotetext{
* Autor para correspondencia.

Correo electrónico: mauricioduquemd@gmail.com (M. Duque-Ramírez).
} 
artery of Adamkiewicz. Its diagnosis is not always possible by visualising its occlusion by computed tomography angiography or by magnetic resonance angiography, since the defects may be evanescent, or there may be a distal compromise with a microvascular obstruction, hardly apparent with selected angiography.

A case is presented of embolic ischaemic myelitis associated with a shaggy aorta, with probable occlusion of the artery of Adamkiewicz being responsible form the acute neurological deterioration of the patient, confirmed by a nuclear magnetic resonance scan.

In order to better describe the features of this disease and to have the appropriate diagnostic and therapeutic strategies for its timely approach, it is considered important to report all similar cases, thus increasing its diagnostic suspicion.

(c) 2019 Sociedad Colombiana de Cardiología y Cirugía Cardiovascular. Published by Elsevier España, S.L.U. This is an open access article under the CC BY-NC-ND license (http:// creativecommons.org/licenses/by-nc-nd/4.0/).

\section{Introducción}

La aterosclerosis es un problema cardiovascular agresivo emergente, asociado a gran morbimortalidad. Se define como aorta shaggy o aorta espiculoanfractuosa a la degeneración ateromatosa extensa de la aorta con superficie friable que predispone a su ulceración difusa con exposición del contenido de la placa y complicaciones embólicas asociadas.

Este compromiso ateromatoso agresivo asociado a embolia recurrente es una condición pobremente esclarecida a pesar de los casos reportados en la literatura mundial, quizá porque las manifestaciones embólicas pueden ser catastróficas y luego de una embolia no siempre puede documentarse su etiología, y por consiguiente no se establece con claridad esta relación causal.

Adicionalmente, hay pacientes con compromiso trombótico difuso y extenso de la aorta que nunca padecen episodios embólicos ${ }^{1}$. Así, pues, debe diferenciarse la embolización de material trombótico desde un aneurisma aórtico que puede resolverse con anticoagulación, trombectomía o reparo del aneurisma, de la ateroembolia desde una aorta extensa y gravemente enferma con múltiples y recurrentes microembolias distales de cristales de colesterol desde sus placas ulceradas donde hay, de hecho, ausencia de trombo como tal' .

Se describe el caso de una paciente que consultó con déficit neurológico motor de las extremidades inferiores y posterior paraplejia espástica, en quien se documentó ateromatosis aórtica grave con síndrome aórtico agudo concomitante (trombo intramural e intraluminal en aorta abdominal) y evidencia de múltiples infartos esplénicos por angiotomografía; la resonancia de columna lumbar contrastada demostró mielopatía con compromiso de la cauda equina, hallazgo que concuerda con mielitis isquémica de probable etiología embólica. Se trata entonces de un caso de aorta shaggy con múltiples manifestaciones embólicas y sospecha de embolización de ateroma hacia la arteria de Adamkiewicz (conocida también como arteria radicular magna y que irriga los dos tercios inferiores de la médula) como etiología de paraplejia mielopática isquémica.

\section{Métodos}

Se hizo una búsqueda no sistemática de la literatura, usando los términos "embolic", "ischemic" y "myelitis" en PubMed, incluyendo términos Mesh (embolism-myletitis). Se halló un reporte de mielopatía secundario a embolia de la arteria braquial. No se encontraron reportes de caso de aorta shaggy como etiología de mielitis isquémica. Se realizó el reporte de caso y su análisis en el marco de la literatura disponible.

\section{Caso}

Paciente femenina, de 70 años de edad, con antecedente de infección por el virus de la hepatitis $B$, cirrosis hepática, hipertensión, hipotiroidismo y extabaquista, con enfermedad renal crónica en estadio 5 , terapia de reemplazo renal con diálisis peritoneal, y adicionalmente falla cardíaca con función sistólica preservada. Había consultado previamente por dolor lumbar intenso, discapacitante, en cuyo estudio habían solicitado de manera ambulatoria radiografía de columna lumbosacra para cuya preparación recibió laxante osmótico con posterior cuadro de deshidratación secundario a enfermedad diarréica aguda de alto gasto, con intolerancia a la vía oral, razón por la cual consultó al servicio de urgencias. Presentó episodio de hipotensión y lipotimia, y durante su recuperación refirió disminución de la fuerza muscular en las extremidades inferiores documentando paraplejia arrefléxica en el examen físico. Inicialmente se sospechó compresión medular neoplásica o por hernia discal. Se realizó resonancia magnética nuclear contrastada de columna lumbar que demostró mielopatía, posiblemente isquémica, que comprometía la cauda equina, hemangioma en T7-T8 y abombamiento discal L3-L4 sin compromiso del canal medular (figs. 1 a 3). Se solicitó angiotomografía de abdomen que evidenció trombo intramural e intraluminal de la aorta abdominal con una extensión longitudinal de $7,5 \mathrm{~cm}$ por encima de los vasos viscerales sin disección de aorta, ateromatosis aórtica severa y aspecto irregular, posiblemente ulcerado de la misma, a nivel torácico sospechoso de aorta shaggy 


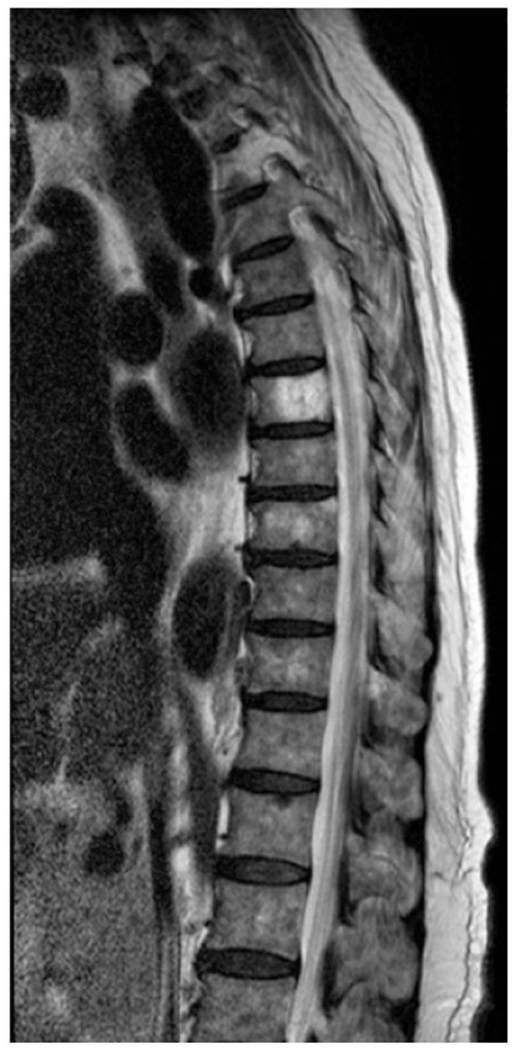

Figura 1 Resonancia magnética nuclear contrastada de columna lumbar en la que se evidenció (de izquierda a derecha) hemangioma en T7-T8, abombamiento discal L3-L4 y mielopatía, posiblemente isquémica, con compromiso de cauda equina.

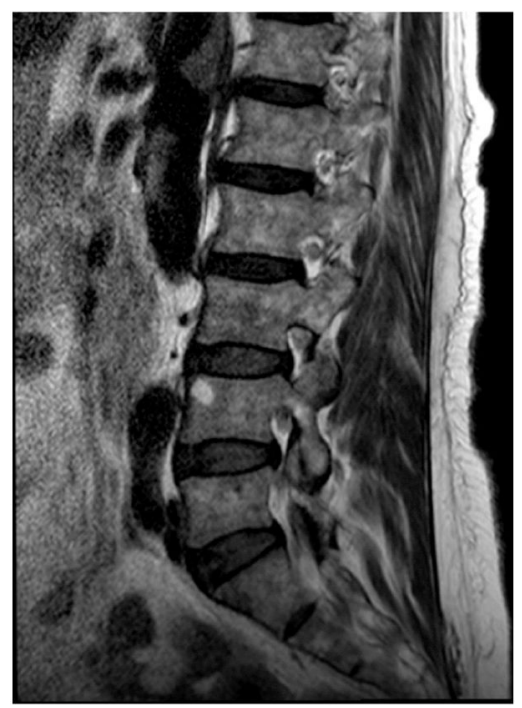

Figura 2 Resonancia magnética nuclear contrastada de columna lumbar en la que se evidenció (de izquierda a derecha) hemangioma en T7-T8, abombamiento discal L3-L4 y mielopatía, posiblemente isquémica, con compromiso de cauda equina.

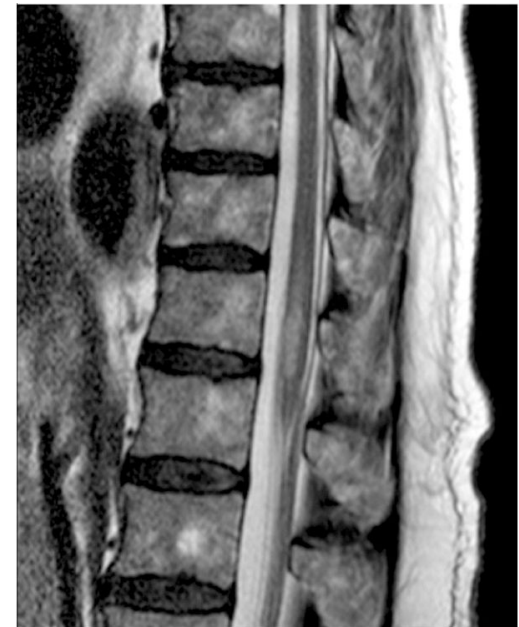

Figura 3 Resonancia magnética nuclear contrastada de columna lumbar en la que se evidenció (de izquierda a derecha) hemangioma en T7-T8, abombamiento discal L3-L4 y mielopatía, posiblemente isquémica, con compromiso de cauda equina.

(fig. 4), con múltiples infartos esplénicos por tomografía (fig. 5). Ante el cuadro neurológico se sospechó ateroembolia de la arteria de Adamkiewicz; sin embargo, no fue posible visualizarla en las imágenes obtenidas (caso llevado a staff de Radiología y Neurocirugía). Se inició manejo con antiagregación dual, sin anticoagulación, con estatinas de alta potencia. Presentó dolor torácico en reposo, por lo que se realizó ecocardiografía de estrés con dobutamina que fue máxima positiva para isquemia en la pared inferior. Fue llevada a coronariografía y se documentó además enfermedad coronaria de dos vasos con oclusión total de la arteria coronaria derecha y lesión en la arteria circunfleja no susceptible de intervencionismo percutáneo. Por múltiples comorbilidades, edad avanzada y pobre red de apoyo familiar se continuó manejo conservador con antiagregación con ácido acetil salicílico, anticoagulación con warfarina y estatinas de alta potencia.

\section{Discusión}

El primer caso de ateroembolia fue descrito por Panum en 1862. Posteriormente fue Kazmier el primero en aclarar que el tipo de material embolizado era relevante al momento de definir el potencial beneficio con la terapia anticoagulante ${ }^{2}$. En 1913, Anitschkow reprodujo un modelo de aterosclerosis aórtica en conejos alimentados con dieta rica en colesterol, estudio pilar para correlacionar los niveles de lipoproteínas plasmáticas con la formación de la placa ateromatosa. Luego, en la década de 1990, Tinuck y Krozon identificaron la aterosclerosis aórtica como fuente de ateroembolia en una serie de pacientes con accidente cerebrovascular, hallazgo cada vez más documentado en pacientes con complicaciones cerebrales isquémicas y manipulación de la aorta ${ }^{3}$.

Se conoce como aorta shaggy a la degeneración vascular y al aspecto de la aorta distorsionado por cristales de colesterol acumulados en la pared vascular. Se denomina síndrome de aorta shaggy a la asociación de estos hallazgos con ateroembolia múltiple ${ }^{4}$; otros nombres que históricamente 


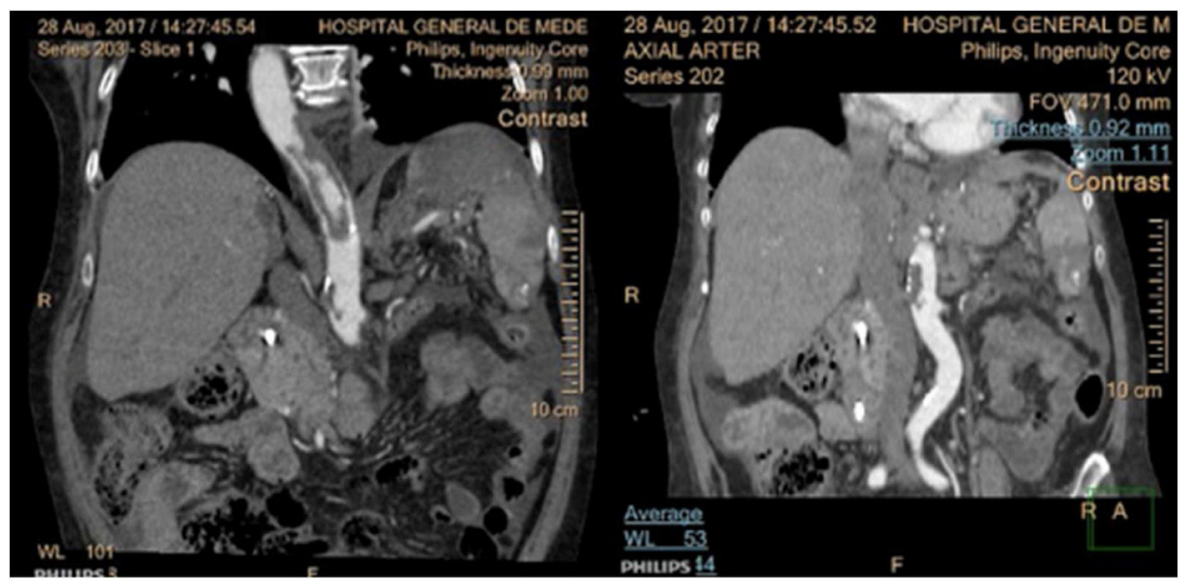

Figura 4 Tomografía de abdomen que muestra trombo intramural e intraluminal al interior de la aorta abdominal en aorta de la unión toracoabdominal, por encima de vasos viscerales, en una extensión longitudinal de $7,5 \mathrm{~cm}$. Se extiende hasta el origen del tronco celíaco sin comprometerlo y se asocia a flap con disección focal.

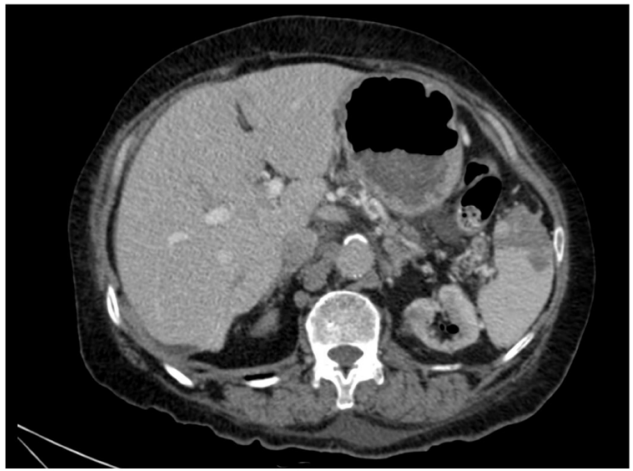

Figura 5 En fase portal de tomografía de abdomen contrastada se observan infartos esplénicos mútiples.

ha recibido esta condición son: aorta en arrecife de coral y aorta hostil ${ }^{3}$. Okada et al. por su parte definieron como aorta shaggy la presencia de ateromas frágiles y espiculados mayores a $5 \mathrm{~mm}$ de grosor en la aorta ascendente y el arco aórtico de acuerdo con hallazgos tomográficos preoperatorios en pacientes en espera de cirugía cardiovascular ${ }^{3}$.

La prevalencia e incidencia de aorta shaggy en poblaciones en riesgo se desconoce, si bien se describen algunos casos de síndrome de aorta shaggy principalmente como complicación de procedimientos endovasculares o quirúrgicos. En sentido estricto, el síndrome de aorta shaggy debería confirmarse cuando ante la presencia clínica de embolia múltiple se demuestra por biopsia la presencia de cristales de colesterol que obstruyen la circulación (arteriolas y arterias pequeñas) de los órganos comprometidos ${ }^{5}$.

Se denomina síndrome del dedo azul a la embolización distal difusa de cristales de colesterol a arterias periféricas, situación que puede ocurrir de manera espontánea o iatrogénica con enfermedad ateromatosa aórtica severa ${ }^{3}$.

La aorta shaggy se asocia a complicaciones como daño renal agudo, pancreatitis, isquemia mesentérica o de extremidades inferiores, entre otras ${ }^{1}$. En la microateroembolia múltiple presente en el síndrome de aorta shaggy se considera que la recurrencia se hace inevitable posiblemente porque una vez producidas las lesiones de la íntima el flujo sanguíneo constante a través del vaso erodado, profundiza el compromiso y perpetúa las embolizaciones del material expuesto del ateroma ${ }^{2}$. El nivel del compromiso no necesariamente predice la distribución anatómica de la embolia pues se considera que el flujo retrógrado en diástole puede favorecer embolia incluso proximal a la lesión dominante ${ }^{2}$, como se ha descrito en algunos casos de enfermedad cerebrovascular.

Hollier et al. describieron una serie de 88 pacientes con aorta shaggy, 36 de los cuales presentaron embolia visceral con daño de órgano, y los 52 restantes embolia del hemicuerpo inferior con compromiso variable desde el nivel glúteo hasta digital ${ }^{2}$. En esta serie se encontró una relación hombre mujer de 3.5 a 1 , pero en el caso del compromiso visceral la relación fue de 11 a 1 . Como regla general los pacientes presentaban múltiples factores de riesgo cardiovascular de manera simultánea (hipertensión arterial, tabaquismo, diabetes mellitus tipo 2), así como eventos cardiovasculares previos (enfermedad coronaria, accidente cerebrovascular). El $27 \%$ de los casos de embolia visceral se consideraron precipitados por intervencionismo endovascular con acceso femoral ${ }^{2}$.

El diagnóstico de aorta shaggy puede hacerse mediante angiotomografía, si bien tiene como desventaja la exposición al medio de contraste y a radiación. Por otra parte, la resonancia magnética contrastada sólo es una alternativa en los casos de alergia al medio de contraste yodado. No se considera la aortografía debido al alto riesgo de complicaciones embólicas. Tampoco se recomienda el uso rutinario de la ecocardiografía transesofágica dado que su alcance es limitado ${ }^{3}$.

En cuanto al manejo médico para el síndrome de aorta shaggy, la terapia con estatinas tiene impacto modesto en la recurrencia de la embolia, mientras que la antiagregación y anticoagulación no la reducen.

Las estatinas se han usado ampliamente para el control de la dislipidemia, así como por sus efectos pleiotrópicos que estabilizan y reducen las placas ateroscleróticas. En estudios observacionales, las estatinas demostraron una reducción absoluta del riesgo del $17 \%$ y una reducción 
relativa del riesgo del 59\% de complicaciones ateroembólicas recurrentes ${ }^{3}$. Nemoto et al. publicaron un estudio retrospectivo en el que se evalúa la regresión de ateromas aórticos torácicos extensos por angiotomografía (definidos como aquellos de grosor mayor a $5 \mathrm{~mm}$, circunferencia comprometida de la aorta torácica mayor al 50\%, extensión mayor a $3 \mathrm{~mm}$ ) valorando el tamaño de la placa antes y después de la intervención con estatinas en 20 pacientes (fluvastatina, simvastatina, pravastatina, pitavastatina, atorvastatina y rosuvastatina) comparado con 7 controles con compromiso aórtico semejante. Se valoró el porcentaje de reducción del radio del ateroma y se halló una reducción de $7,1 \% \pm 6,4 \%$, diferencia estadísticamente significativa respecto al grupo de control $(p<0,01)$ Siontis et al., «Transcatheter aortic valve implantation vs. surgical aortic valve replacement for treatment of severe aortic stenosis: A meta-analysis of randomized trials»; Reardon et al., «Surgical or Transcatheter Aortic-Valve Replacement in Intermediate-Risk Patients»; Thyregod et al., "Transcatheter versus surgical aortic valve replacement in patients with severe aortic valve stenosis: 1year results from the all-comers NOTION randomized clinical trial» ${ }^{6}$.

De otro lado, no hay evidencia sobre la eficacia de la terapia antiplaquetaria y se considera que la anticoagulación podría incluso estar contraindicada pues podría favorecer la inestabilidad de los trombos murales, limitar la cicatrización de las úlceras y así exacerbar el riesgo embólico de los cristales de colesterol al interior de las mismas ${ }^{1-5}$.

De la revisión de la literatura disponible no puede concluirse que exista superioridad del intervencionismo endovascular o abordaje quirúrgico sobre el manejo conservador. Se han descrito incluso técnicas mixtas donde la exclusión del segmento enfermo con prótesis endovascular no siempre es posible teniendo en cuenta que el compromiso aórtico puede extenderse a su porción torácica o suprarrenal lo que dificulta el intervencionismo percutáneo ${ }^{1}$. La exclusión endovascular del segmento enfermo es riesgosa pues puede inducir ateroembolia iatrogénica a cualquier nivel. El riesgo de embolia durante reparo quirúrgico de la aorta es muy elevado. Kwon et al. describieron en su estudio observacional retrospectivo que el $11 \%$ de los pacientes llevados a reparo quirúrgico de aneurisma de aorta abdominal (AAA) tenían aorta shaggy, y que presentarla aumentaba 4,1 veces la morbimortalidad a 30 días en comparación con los pacientes con AAA aislada (IC 95\% 1,7-9,7; p 0,002) ${ }^{7}$. La manipulación quirúrgica puede desprender material de la placa con embolia sistémica "en ducha", potencialmente amenazante para la vida (compromiso visceral, mesentérico, renal, glútea, así como síndrome del dedo azul que puede amenazar la viabilidad de la extremidad $)^{4}$.

Existen reportes en la literatura sobre diferentes técnicas empleadas en el abordaje quirúrgico de la aorta shaggy que pretenden la menor incidencia de embolia sistémica posible; en la literatura se han descrito procedimientos combinados que incluyen el uso concomitante de filtros arteriales, que al parecer ha tenido éxito en la reducción de las complicaciones ateroembólicas ${ }^{8-13}$. Los puentes axilofemorales o aortobifemorales con exclusión de las arterias ilíacas se han contemplado históricamente como una alternativa cuando el compromiso toracoabdominal es extenso ${ }^{1}$.
La arteria de Adamkiewicz es un vaso de pequeño calibre que nace de las arterias intercostales o lumbares que se derivan de la aorta. Se caracteriza por tener lateralización en posiciones anatómicas variables desde T5 y hasta L2. Su detección imagenológica representa un reto y es invariablemente difícil, identificándose en solo $20 \%$ de las tomografías en fase arterial en el mejor de los casos $^{14}$. Adicionalmente, la oclusión de esta arteria no es de fácil visualización por angiotomografía ni por angiorresonancia, posiblemente por evanescencia del émbolo o por compromiso distal microvascular laboriosamente aparente aun con arteriografía selectiva. Hay estudios que han comparado la tomografía de alta resolución (con cortes de $0,5 \mathrm{~mm}$ ) con la tomografía de ultra alta resolución (con cortes de $0,25 \mathrm{~mm}$ ) demostrando que mientras más finos sean los cortes mejor será el rendimiento de la técnica en la visualización de la arteria radicular magna ${ }^{15}$.

Retomando el caso que atañe a este reporte, es importante recordar que la mielitis isquémica embólica se asocia principalmente a embolia fibrocartilaginosa desde núcleos pulposos. No hay reportes que asocien ateroembolia aórtica por aorta shaggy con obstrucción de la arteria de Adamkiewicz.

\section{Conclusión}

La aorta shaggy es una manifestación infrecuente pero grave de la ateromatosis aórtica, asociada a riesgo ateroembólico masivo y recurrente con desenlaces clínicos usualmente catastróficos. Grandes interrogantes respecto del manejo médico e intervencionista/quirúrgico persisten sin resolver. En nuestro conocimiento este es el primer reporte de mieliopatía isquémica ateroembólica asociada a aorta shaggy con posible oclusión de la arteria de Adamkiewicz.

\section{Financiación}

Ninguna.

\section{Conflicto de intereses}

Ninguno.

\section{Bibliografía}

1. Giulio I, Bresadola L, D’Urso A, Ceccanei G, Vietri F. Simultaneous Stent Grafting of the Descending Thoracic Aorta and Aortofemoral Bypass for "Shaggy Aorta"' Syndrome. Canadian J Surg. 2007;50:E1-2.

2. Larry H, Kazmier FJ, Ochsner J, Bowen JC, Procter D. Shaggy" Aorta Syndrome with Atheromatous Embolization to Visceral Vessels. Ann Vasc Surg. 1991;5:439-44, https://doi.org/10.1007/BF02133048

3. Fukuda, Ikuo, Kazuyuki Daitoku. Masahito Minakawa, y Wakako Fukuda. Shaggy and Calcified Aorta: Surgical Implications». Gen Thorac Cardiovasc Surg. 2013;61:301-13, https: / /doi.org/10.1007/s11748-013-0203-y.

4. Wada, Tomoyuki, Hirofumi Anai, Takashi Shuto, Takeshi Sakaguchi, Tetsuo Hongo. Ret al. A New Method for Protection from Shower Embolism during TEVAR on a 
Shaggy Aorta. GenThorac Cardiovasc Surg. 2015;63:105-8, https://doi.org/10.1007/s11748-013-0278-5.

5. Hayashida, Naoki, Hirokazu Murayama, Yoko Pearce, Souichi Asano, Yukio Ohashi, et al. Shaggy Aorta Syndrome After Acute Arterial Macroembolism: Report of a Case. Surg Today. 2004;34:354-6, https://doi.org/10.1007/s00595-003-2694-0.

6. Nemoto, Masaru, Katsuyuki Hoshina, Toshio Takayama, Sumio Miura, Tatsu Nakazawa, et al. Statins Reduce Extensive Aortic Atheromas in Patients with Abdominal Aortic Aneurysms. Ann Vasc Dis. 2013;6:711-7, https://doi.org/10.3400/avd.oa.1300065

7. Kwon $\mathrm{H}$, Han $\mathrm{Y}$, Noh M, Gwon JG, Cho YP, Kwon TW. Impact of Shaggy Aorta in Patients with Abdominal Aortic Aneurysm Following Open or Endovascular Aneurysm Repair. Eur J Vasc and Endovasc Surg. 2016;52:613-9, https://doi.org/10.1016/j.ejvs.2016.08.010.

8. Hori, Daijiro, Koichi Yuri, Kenichiro Noguchi, Yohei Nomura, Hiroyuki, Tanaka. Case Report: Successful Endovascular Treatment of Saccular Thoracic Aneurysm with "Shaggy Aorta" Ann Thorac Cardiovasc Surg. Official Journal of the Association of Thoracic and Cardiovascular Surgeons of Asia. 2012;18:536-9.

9. Takagi, Yasushi, Motomi Ando, Kiyotoshi Akita, Michiko Ishida, Kan Kaneko, Masato Sato. Arch Replacement Using Antegrade Selective Cerebral Perfusion for Shaggy Aorta. Asian Cardiovascular and Thoracic Annals. 2013;21:31-6, https://doi.org/10.1177/ 0218492312446205

10. Takano, Tomohiro, Shinya Takase, Akihito Kagoshima, Hitoshi, Yokoyama. Prevention of Embolization in Concomitant Endovascular Therapy for Thoracic and Abdominal Aneurysms with Severely Atheromatous Aorta. Ann Vasc Dis. 2016:345-8, https://doi.org/10.3400/avd.cr.16-6 0006
11. Igarashi, Takashi, Shinya Takase, Hirono Satokawa, Yukitoki Misawa, Hiroki Wakamatsu, et al. Thoracic Endovascular Aortic Repair with Visceral Arteries Intermittent Clamp Technique for Descending Thoracic Aortic Aneurysm with Shaggy Aorta. Ann Vasc Surg. 2013;27:974, e11-974.e14. https: / /doi.org/10.1016/j.avsg.2013.02.006 pdf

12. Tanaka, Satofumi, Kuniyoshi Tanaka, Koichi Morioka, Narihisa Yamada, Atsushi Takamori, et al. Thrombosed Abdominal Aortic Aneurysm Associated with an Extensively "Shaggy" Aorta Repaired Anatomically via a Thoracoabdominal Approach with Supraceliac Aortic Clamping: Report of a Case. Surgery Today. 2010;40:866-70, https://doi.org/10.1007/s00595-0084115-x

13. Murakami, Takashi, Yohei Kawatani, Yoshitsugu Nakamura, Takaki Hori. Transapical Endovascular Aortic Aneurysm Repair in a Patient with Shaggy Aorta Syndrome. Ann Vasc Surg. 2015;29, 838.e7-838.e9. https://doi.org/10.1016/j.avsg.2014. 11.017

14. Guziński, Maciej, Maciej Bryl, Katarzyna Ziemińska, Kamila Wolny, Marek Sąsiadek, Jerzy S. Garcarek. Detection of the Adamkiewicz Artery in Computed Tomography of the Thorax and Abdomen. Advances in Clinical and Experimental Medicine: Official Organ Wroclaw Medical University. 2017;26: 31-7.

15. Yoshioka, Kunihiro, Ryoichi Tanaka, Hidenobu Takagi, Yuta Ueyama, Kei Kikuchi, et al. Ultra-High-Resolution CT Angiography of the Artery of Adamkiewicz: A Feasibility Study. Neuroradiology. 2018;60:109-15, https://doi.org/10.1007/s00234017-1927-7 